\title{
Flora béntica y reproducción de las algas Batophora spp. (Chlorophyta: Dasycladaceae) de una laguna costera contaminada (Bahía de Chetumal, México)
}

\author{
L.I. Quan-Young ${ }^{1}$, S.G. Jiménez-Flores \& J. Espinoza-Ávalos \\ ECOSUR, Apartado Postal 424, Chetumal, Quintana Roo 77000, México; lquan@ecosur-qroo.mx, Fax: \\ 52(983)8350454
}

Recibido 13-XII-2000. Corregido 13-XI-2001. Aceptado 27-II-2003.

\begin{abstract}
Abstrac. Benthic flora and reproduction of Batophora spp. algae (Chlorophyta: Dasycladaceae) in a polluted coastal lagoon (Chetumal Bay, Mexico). The benthic flora, and the vegetative and reproductive characters of the algae Batophora oerstedii and B. occidentalis (Chlorophyta) were recorded from five sites of Chetumal Bay, Quintana Roo, Mexico. A sewage gradient has been reported along those sites. Plants were sampled in May and October 1999, which corresponded to dry and rainy seasons, respectively. Forty taxa were found, 11 are new records for the Chetumal Bay, and 6 are new records for the Mexican Caribbean. Enteromorpha species were present in sites known as rich in organic matter (both from anthropogenic and natural sources). Batophora spp. is the dominant algae in all Chetumal Bay. However, it was absent next to sewage outfalls. The morphological characters of $B$. oerstedii and $B$. occidentalis did not change significantly along the sites reported as polluted. The length and width of gametophores, as well as the diameter of the gametangia were clearly different for both species. Different reproductive strategies may help B. oerstedii and B. occidentalis to closely coexist in the Chetumal Bay. Rev. Biol. Trop. 54(2): 341-355. Epub 2006 Jun 01.
\end{abstract}

Key words: Enteromorpha, benthic flora, pollution, Caribbean, Yucatan Peninsula, coexistence.

La vegetación acuática de la Bahía de Chetumal es una de las más pobres de las aguas costeras de Quintana Roo, de acuerdo a los resultados de Huerta-Múzquiz et al. (1987). Sin embargo, puede proveer parte de la dieta que mantiene a la población de manatí Trichechus manatus L. que ahí habita (AxisArroyo et al. 1998). También proporciona alimento y refugio a invertebrados, los cuales a su vez son consumidos por otros organismos, como los peces. Es decir, las macroalgas son un componente importante de la comunidad biótica de la Bahía de Chetumal.

Las macroalgas que dominan en la Bahía de Chetumal son Batophora oerstedii J. Agardh y B. occidentalis (Harv.) S. Berger \& Kaever ex M.J. Wynne. Ambas coexisten muy cercanamente, compartiendo siempre las mismas rocas como sustrato (Gómez-Poot et al., 2002).
Sin embargo, frente a la parte urbana de la bahía se han registrado especies de Enteromorpha y Polysiphonia (Huerta-Múzquiz y GarzaBarrientos 1980), las cuales en otras zonas del mundo se registran como especies indicadoras de contaminación orgánica (Murray y Littler 1978, Druehl 1981, Levine 1984, Guangzhou 1987, Aguilar-Rosas y Pacheco-Ruíz 1989, Morand y Brand 1996). En otras ocasiones el efecto de la contaminación se ha reportado como una reducción en el número de especies (Murray y Littler 1978, Neverauskas 1987, Cecere et al. 1991).

En la Bahía de Chetumal se vierten aguas residuales municipales sin recibir ningún tratamiento previo. A ese cuerpo de agua también llegan diferentes fertilizantes y pesticidas, provenientes principalmente de actividades relacionadas al cultivo de la caña de azúcar. 
Chavira-Martínez et al. (1992) y Ortíz y Sáenz (1995) informaron que los valores de materia orgánica en los sedimentos fueron mayores frente a la desembocadura del Río Hondo y de la ciudad de Chetumal, y que disminuían al alejarse de esos puntos. Ortiz y Sáenz (1997) registraron una tendencia similar para detergentes, y determinaron que se presentaban concentraciones de bacterias coliformes fecales que rebasaban los valores permisibles por la legislación mexicana (Ortíz y Sáenz 1999).

En este estudio se trazaron los siguientes objetivos: 1) determinar si la presencia o ausencia de vegetación acuática reflejaba la contaminación orgánica (del sitio 1 , frente a la ciudad de Chetumal, al 5; Fig.1), 2) efectuar mediciones de partes vegetativas y reproductivas de Batophora occidentalis y B. oerstedii, para determinar si existían evidencias de cambios morfológicos en el gradiente mencionado, 3) determinar si alguna de esas dos especies podrían considerarse como especie indicadora de contaminación orgánica, y 4) analizar si las mediciones biológicas pueden ayudar a explicar la coexistencia de las dos especies en el sistema estuarino de la Bahía de Chetumal. Adicionalmente, se analiza información florística obtenida en 1991, para obtener una visión más amplia de la vegetación de esta laguna costera.

\section{MATERIALES Y MÉTODOS}

Área de estudio: La Bahía de Chetumal se ubica en la parte sur de Quintana Roo y es parte de la zona fronteriza entre México y Belice. Esta limitada por los paralelos $18^{\circ} 21^{\prime}$ y $18^{\circ} 52^{\prime} \mathrm{N}$ y los meridianos $87^{\circ} 54^{\prime}$ y $88^{\circ} 23^{\prime} \mathrm{W}$. Tiene una superficie de $1098 \mathrm{Km}^{2}$, anchura máxima de $20 \mathrm{Km}$, longitud de $67 \mathrm{Km}$ y con una sección SE que abre al Mar Caribe. Los principales aportes de agua provienen de los ríos Hondo, Crick y Nuevo, de las lagunas Guerrero y Shipstern, de ríos subterráneos y de agua marina.

La Bahía de Chetumal es un sistema lagunar estuarino semicerrado con influencia

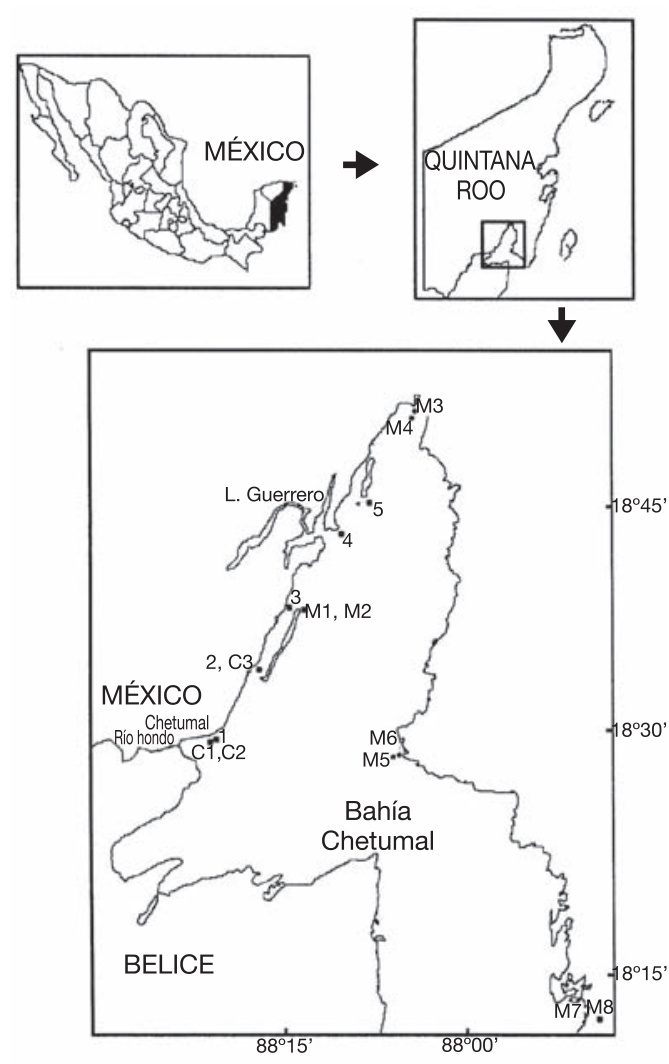

Fig. 1. Zona de estudio y sitios donde se recolectó la vegetación béntica en octubre de 1991 (M1-M8), mayo y octubre de 1999 (1-5) y marzo de 2000 (C1-C3) (ver Materiales y Métodos).

Fig. 1. Study zone, and sites where benthic vegetation was sampled on October 1991 (M1-M8), May and October 1999 (1-5) and March 2000 (C1-C3) (see Material and Methods).

marina limitada, hipohalino por no presentar salinidades promedio mayores a $14 \mathrm{ppm}$, somero con una profundidad media de $3.2 \mathrm{~m}$. Las estaciones de muestreo fueron seleccionadas con base en la intensidad de urbanización.

\section{Recolecta de organismos y trabajo de} laboratorio. Se realizaron tres muestreos; en mayo (20 y 21) y Octubre (20, 21 y 27) de 1999 y en marzo de 2000. Las plantas de Batophora spp. Se obtuvieron de rocas localizadas a $1 \mathrm{~m}$ de profundidad, a $30 \mathrm{~m}$ de la orilla, y de 5 sitios: 1. Nieves, 2. Alacranes, 3. Luis Echeverría, 4. 
La Barra y 5. Cayo Venado (Fig. 1). En cada sitio también se realizó una recolecta general de macroalgas en las dos primeras fechas mencionadas; tanto en la orilla, como a $1 \mathrm{~m}$ de profundidad. Las plantas se separaron de las rocas con un cuchillo, colocadas en bolsas de plástico y preservadas en agua de la bahía con formol al $4 \%$.

En el primer muestreo se recolectaron todas las plantas de Batophora spp. encontradas en cinco rocas. En el laboratorio se tomaron al azar 200 plantas y se registró el número de plantas vegetativas y en reproducción de $B$. occidentalis (con gametóforos ovoides) y $B$. oerstedii (con gametóforos esféricos). Posteriormente se midió su largo, y de las que se encontraban en reproducción se tomaron 60 plantas de cada especie; entre ellas se separaron las que presentaban gametóforos maduros e inmaduros (en los inmaduros el contenido celular del gametóforo se encontraba difuso, mientras que en los maduros se encontraba delimitado en gametangios bien diferenciados). Se tomaron 40 plantas con gametóforos maduros de cada especie y se midió el largo y ancho de un gametóforo por planta. Esos mismos gametóforos fueron rotos con pinzas de relojero y se midió el diámetro de uno de los gametangios liberados (terminología reproductiva de acuerdo a Berger y Kaever 1992).

Para el segundo muestreo, en cada sitio se extrajeron al azar 4-5 rocas, de donde se cosecharon las plantas de Batophora spp. que se encontraron en 15 cuadrantes de $3 \times 3 \mathrm{~cm}$. En el laboratorio se registró el número de plantas vegetativas y en reproducción. De las que se encontraron en reproducción se midió su largo y se registró aquellas pertenecientes a $B$. occidentalis y $B$. oerstedii. Luego se separaron entre las que presentaron gametóforos maduros e inmaduros. De las plantas con gametóforos maduros se midió el largo y el ancho de un gametóforo por planta, lo mismo que el diámetro de un gametangio, como se indicó antes.

La tercera recolecta incluyó mediciones de biomasa fresca en los cinco sitios, además de otros tres que correspondieron a $\mathrm{C} 1$ : desagüe ubicado frente al Congreso del Estado, C2: $\approx 30$ $\mathrm{m}$ al Norte de C1, y C3: en Alacranes, $\approx 25 \mathrm{~m}$ al Norte del sitio 2. En los ocho sitios se cosecharon las plantas que se encontraron en 10 cuadrantes de $5 \times 5 \mathrm{~cm}$, sobre rocas de la orilla.

Adicionalmente se analizó otra serie de muestras recolectadas en Octubre de 1991, en 8 sitios de la bahía de Chetumal: M1. Isla Tamalcab (punta Norte, raíces de mangle), M2. Isla Tamalcab (punta Norte, arena), M3. Entrada Río Crick, M4. Ojo de agua 1, M5. Ojo de agua 2, M6. Punta Calentura, M7. Bacalar Chico (entrada, lado Sur), M8. Bacalar Chico (lado Norte) (Fig. 1). Las muestras fueron separadas, luego identificadas con la ayuda de publicaciones como las de Taylor (1960), Joly (1967) y Ortega (1984). Para el arreglo sistemático de las especies se siguió a Ortega (1984) y Wynne (1998). Para la asignación de taxa como nuevos registros se revisaron los trabajos de Huerta-Múzquiz y Garza-Barrientos (1980), Huerta-Múzquiz et al. (1987), OrtegónAznar (1997) y Dreckmann (1998).

Datos ambientales y análisis estadísticos. Valores mensuales promedio de temperatura ambiental, precipitación pluvial, así como de velocidad y dirección predominante del viento de la ciudad de Chetumal se obtuvieron de la Comisión Nacional del Agua, Subdelegación Quintana Roo.

Los valores promedio obtenidos de Batophora spp. se analizaron estadísticamente mediante el programa Statistica ${ }^{\circledR}$, versión 4.3 para Windows (Statsoft, Inc., Tusla, USA). Los resultados no tuvieron una distribución normal, ni homogeneidad de varianzas, por lo que los análisis se realizaron con estadística no paramétrica, utilizando el modelo Kruskal-Wallis. Los valores promedio y los errores estándar se graficaron utilizando el programa Statistica ${ }^{\circledR}$.

El agrupamiento por similitud de especies, con respecto a los sitios, para las tres fechas de muestreo, se analizó con el programa ANACOM (de la Cruz 1994). Para ello se utilizó el índice de Jaccard, Modo ø (columnas), unión medio no ponderada, con la cual se obtuvieron las entidades y el nivel de similitud entre sitios. 


\section{RESULTADOS}

Se identificaron 42 taxa de vegetación béntica, repartidas en 6 microalgas, 34 macroalgas, y 2 fanerógamas (Cuadro 1 y 2). La mayor riqueza se presentó en Bacalar Chico (sitios M7 y M8, 13 taxa), mientras que la menor ocurrió en Punta Calentura (sitio M6, 2 taxa); ambas localidades fueron visitadas en 1991. El número de taxa fue el mismo para los sitios 1,2 y 5 en los dos muestreos de 1999 (5, 8 y 6 taxa, respectivamente). El número de taxa fue variable (entre 5 y 9) para los sitios 3 y 4 (Cuadro 1).

Se obtuvieron 11 nuevos registros de vegetación béntica para la Bahía de Chetumal: 5 Rhodophyta, 5 Chlorophyta, y 1 Chromophyta, (Cuadro 1). Entre estos se incluyen 6 que representan nuevos registros para Quintana Roo (Cuadro 1).

La similitud de la vegetación béntica en mayo y octubre de 1999 presentaron dos entidades con niveles mayores a 0.5 , mientras que el sitio 1 quedó separado de los otros cuatro sitios (Fig. 2a y 2b).

Los valores promedio de talla de Batophora occidentalis y $B$. oerstedii mostraron diferencia estadística significativa $(p<0.0001)$ entre el mes de mayo y octubre, en el primer mes las plantas fueron más grandes que en el segundo. Sin embargo, la talla de las plantas en reproducción entre las dos especies no presentó diferencias estadísticas significativas $(\mathrm{p}>0.05)$ ni en mayo ni en Octubre (Fig. 3a y 3b). Por otra parte, en mayo (Fig. 3a) las plantas en reproducción de las dos especies fueron significativamente mayores $(\mathrm{p}<0.05)$ a las plantas vegetativas, lo cual no ocurrió en Octubre (Fig. $3 \mathrm{~b}$ ), cuando ambos tipos de plantas fueron similares en tamaño $(\mathrm{p}>0.05)$.

El número de plantas en reproducción de Batophora occidentalis para el mes de mayo presentó el mayor número de plantas en reproducción (47) en el sitio $5 \mathrm{y}$ el menor número en el sitio 2 (16), mientras que $B$. oerstedii presentó el mayor número de plantas en reproducción en el sitio 1 (54) y el menor en el sitio 3 (17) (Fig. 4a). Para el mes de octubre, $B$. occidentalis presentó el mayor número de plantas en reproducción en los sitios 1 y 2 (59 y 61 , respectivamente) y el menor número en el sitio 5 (12), mientras que $B$. oerstedii presentó el mayor número de plantas en reproducción en el sitio 3 (44) y el menor en el sitio 5 (1) (Fig. $4 b)$. Esto es, existieron diferentes respuestas reproductivas de las dos especies en los sitos de estudio. En general, la proporción de plantas inmaduras de ambas especies fue mayor que las maduras para el mes de mayo que para el mes de octubre (Fig. 4a y 4b). En octubre fue evidente una diferencia en el ciclo reproductivo entre ambas especies; en este caso, con un mayor número de plantas reproductivas para $B$. occidentalis, que para $B$. oerstedii, con excepción en el sitio 3 (Fig. 4b). De manera similar, en este mes también se evidenciaron
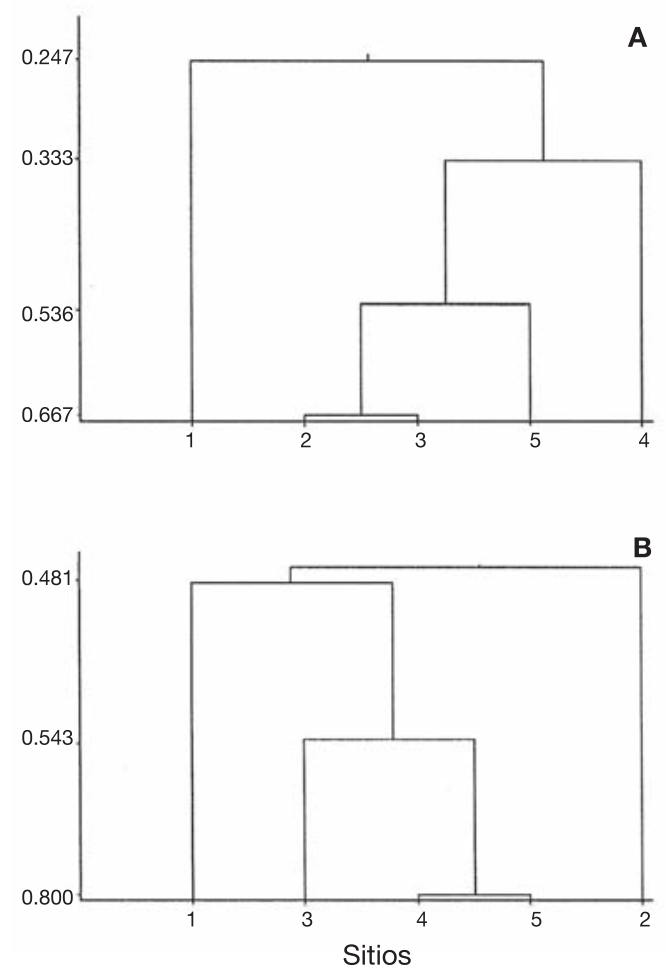

Fig. 2. Agrupamiento de la vegetación béntica para cinco sitios de la Bahía de Chetumal, en mayo (Fig. 2a) y octubre (Fig. 2b) de 1999.

Fig. 2. Clusters of benthic vegetation from five sites of the Chetumal Bay, during May (Fig. 2a) and October 1999 (Fig. 2b). 


\section{CUADRO 1}

Lista sistemática de la vegetación béntica de la Bahía de Chetumal, Quintana Roo, recolectada en 1991 (Octubre, sitios M1-M8) y 1999 (Mayo y octubre, sitios 1-5)

TABLE 1

Systematic checklist of benthic vegetation from the Chetumal Bay, State of Quintana Roo, México, sampled on 1991 (October, sites M1-M8) and 1999 (May and October, sites 1-5)
M1
M2 M3 M4 M5 M6
M7 M8
23
4

$\begin{array}{llll}1 & 2 & 3 & 4\end{array}$

\section{MICROALGAS}

Rhodophyta

Bangiophycidae

Goniotrichales

Goniotrichaceae

Chroodactylon sp.

Heterokontophyta

Bacillariophyceae

Pennatophycidae

Achanthaceae

Cocconeis sp.

Naviculales

Naviculaceae

Cymbella sp.

Navicula sp.

Pinnularia sp.

Epithamiaceae

Epithemia sp.

\section{MACROALGAS}

Chromophyta

Xanthophyceae

Vaucheriales

Vaucheriaceae

**Vaucheria sp.

X X

X

Cyanophyta

Oscillatoriales

Oscillatoriaceae

Lyngbya sp.

X

Phomidiaceae

Symploca sp. 
CUADRO 1 (Continued)

Lista sistemática de la vegetación béntica de la Bahía de Chetumal, Quintana Roo, recolectada en 1991 (Octubre, sitios M1-M8) y 1999 (Mayo y octubre, sitios 1-5)

TABLE 1 (Continued)

Systematic checklist of benthic vegetation from the Chetumal Bay, State of Quintana Roo, México, sampled on 1991 (October, sites M1-M8) and 1999 (May and October, sites 1-5)

$\begin{array}{llllllllllllllllll}\text { M1 } & \text { M2 } & \text { M3 } & \text { M4 } & \text { M5 } & \text { M6 } & \text { M7 } & \text { M8 } & 1 & 2 & 3 & 4 & 5 & 1 & 2 & 3 & 4 & 5\end{array}$

Rhodophyta

Florideophycidae

Nemaliales

Lemaneaceae

Lemanea $\mathrm{sp}$.

X

X $\quad$ X $\quad$ X $\quad$ X

Gigartinales

Caulecanthaceae

Catenella

caestepitosa (Wither.)

L.M. Irvine in Parke

X X X

$\begin{array}{llll}\mathrm{X} & \mathrm{X} & \mathrm{X}\end{array}$

\& Dixon

Hypneaceae

Hypnea spinella

(C. Agardh) Kützing

X

Ceramiales

Ceramiaceae

Ceramium sp.

X

Seirospora sp.

$\mathrm{X}$

Delesseriaceae

**Cotoniella

sanguinea Howe

X

Rhodomelaceae

Bostrychia montagnei

Harvey

X X X

X X

**B. moritziana

(Sonder ex Kützing)

J. Agardh

**B. pilulifera

Montagne

B. tenella (J.V.

Lamouroux) J.

Agardh

B. radicans

(Montagne)

$x$

X

Montagne in Orbigny

Digenea simplex

(Wulfen) C. Agardh

X

X X

$\begin{array}{llll}X & X & X & X\end{array}$

$\begin{array}{llllllllllllllllll}X & X & X & X & X & X & X & X & & X\end{array}$

$\mathrm{X}$

$\mathrm{X}$ 
CUADRO 1 (Continued)

Lista sistemática de la vegetación béntica de la Bahía de Chetumal, Quintana Roo, recolectada en 1991 (Octubre, sitios M1-M8) y 1999 (Mayo y octubre, sitios 1-5)

TABLE 1 (Continued)

Systematic checklist of benthic vegetation from the Chetumal Bay, State of Quintana Roo, México, sampled on 1991 (October, sites M1-M8) and 1999 (May and October, sites 1-5)

$\begin{array}{llllllllllllllllll}\text { M1 } & \text { M2 } & \text { M3 } & \text { M4 } & \text { M5 } & \text { M6 } & \text { M7 } & \text { M8 } & 1 & 2 & 3 & 4 & 5 & 1 & 2 & 3 & 4 & 5\end{array}$

Laurencia obtusa

(Hudson) J.V.

X

Lamouroux

*Polysiphonia

denudata (Dillwyn)

Greville ex Harvey

X

in Hook

**P. ramentaceae

Harvey

X

P. sphaerocarpa

Børgesen

P. subtilissima

Montagne

X

X X

X

X

Chlorophyta

Charales

Charophyceae

**Chara sp.

X X

X

X X

Ulvales

Ulvaceae

Enteromorpha

compressa (Linnaeus)

Nees

X

X X

E. flexuosa (Wulfen)

J. Agardh

Enteromorpha sp.

X

Anadyomenaceae

* Anadyomene stellata

(Wulfen in Jacq.) X

C. Agardh

Boodleaceae

Boodlea sp.

Siphonocladaceae

Cladophoropsis

membranaceae

(C. Agardh) Børgesen

X

X X

X X 
CUADRO 1 (Continued)

Lista sistemática de la vegetación béntica de la Bahía de Chetumal, Quintana Roo, recolectada en 1991 (Octubre, sitios M1-M8) y 1999 (Mayo y octubre, sitios 1-5)

TABLE 1 (Continued)

Systematic checklist of benthic vegetation from the Chetumal Bay, State of Quintana Roo, México, sampled on 1991 (October, sites M1-M8) and 1999 (May and October, sites 1-5)

$\begin{array}{llllllllllllllllll}\text { M1 } & \text { M2 } & \text { M3 } & \text { M4 } & \text { M5 } & \text { M6 } & \text { M7 } & \text { M8 } & 1 & 2 & 3 & 4 & 5 & 1 & 2 & 3 & 4 & 5\end{array}$

*Dictyosphaeria ocellata (M. Howe)

X X

J.L. Olsen

Bryopsidales

Bryopsidaceae

*Derbesia sp.

X

Udoteaceae

*Boodleopsis pusilla

(Collins) W.R. Taylor,

X

A.B. Joly \& Bernat

Penicillus capitatus

Lamouroux

X

Dasycladales

Dasycladaceae

Batophora oerstedii

J. Agardh

X X

X

$\begin{array}{lllllllllll}X & X & X & X & X & X & X & X & X & X & X\end{array}$

\section{FANEROGAMAS}

Magnoliophytae

Najadales

Cymodoceaceae

Halodule wrightii

Ascherson

$\mathrm{X}$

$\mathrm{X}$

Potamogetonaceae

Ruppia maritima

Linnaeus

$\begin{array}{llllllllllllllllllllllll}\text { TOTAL DE TAXA } & 3 & 6 & 4 & 3 & 8 & 2 & 13 & 5 & 5 & 9 & 5 & 9 & 5 & 6 & 8 & 9 & 5 & 6\end{array}$

Nota: Los taxa con un asterisco representan nuevos registros para la Bahía de Chetumal; aquellos con dos representan además nuevos registros para el Caribe mexicano.

Note: Taxa with an asterisk represent new records for the Chetumal Bay; those with two asterisks are new records for the Mexican Caribbean. Octubre de 1991 Mayo de 1999 Octubre de 1999. 

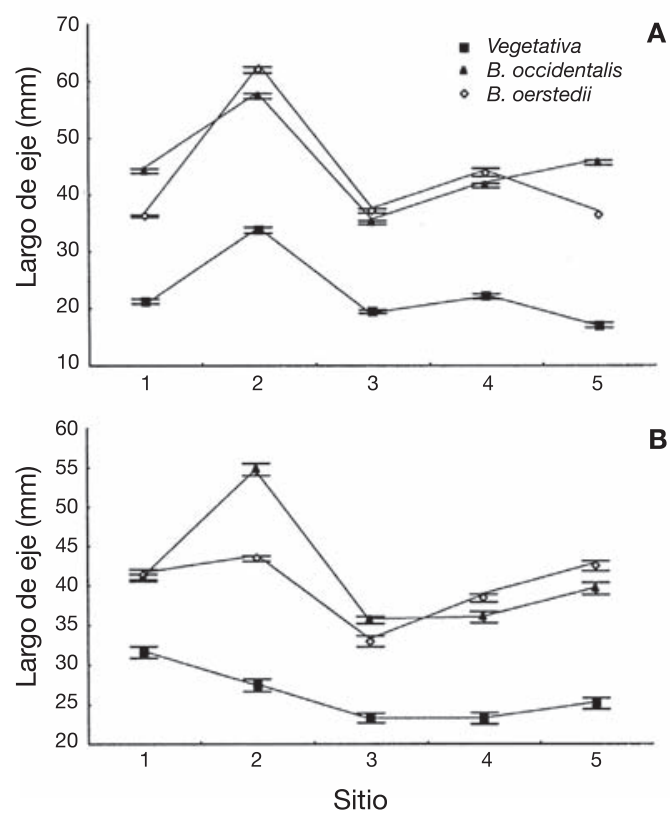

Fig. 3. Largo de eje (mm) de Batophora occidentalis y B. oerstedii en cinco sitios de la Bahía de Chetumal, durante mayo (Fig. 3a) y octubre (Fig. 3b) de 1999.

Fig. 3. Length of the axis ( $\mathrm{mm}$ ) of Batophora occidentalis and $B$. oerstedii in five sites from the Chetumal Bay, during May (Fig. 3a) and October (Fig. 3b) of 1999.

diferencias entre plantas reproductivas maduras e inmaduras de las dos especies. Por ejemplo, en el sitio 2, B. occidentalis presentó una proporción similar de plantas maduras e inmaduras, mientras que $B$. oerstedii presentó una mayor proporción de plantas maduras que inmaduras (Fig. 4b). Esto es, las dos especies de Batophora presentaron estrategias reproductivas diferentes bajo las mismas condiciones estuarinas de Bahía Chetumal.

El largo de gametóforos de las plantas de Batophora occidentalis y B. oerstedii (Fig. 5), fue significativamente diferente $(\mathrm{p}<0.0001)$ entre los sitios de colecta para el mes de mayo; mayor en sitios alejados de la ciudad de Chetumal (ubicada frente al sitio 1). Sin embargo, en Octubre el largo de gametóforos no fue significativamente diferente $(\mathrm{p}>0.05)$ entre los sitios de colecta, para ambas especies. El largo del gametóforo de ambas especies no mostró
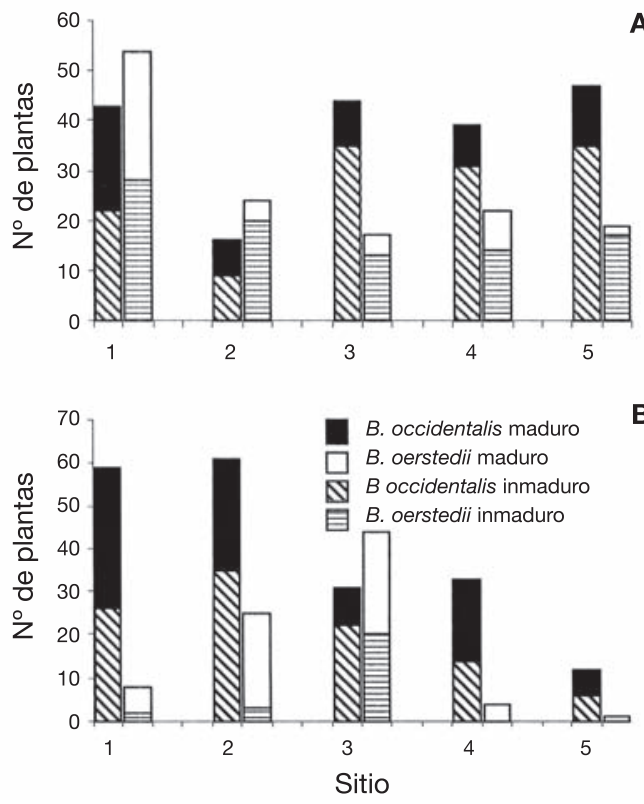

Fig. 4. Número de plantas en reproducción de Batophora occidentalis y B. oerstedii, en cinco sitios de la Bahía de Chetumal, durante mayo (Fig. 4a) y octubre (Fig. 4b) de 1999.

Fig. 4. Number of reproductive plants of Batophora occidentalis and $B$. oerstedii in five sites from the Chetumal Bay, during May (Fig. 4a) and October (Fig. 4b) of 1999.

diferencias significativas $(\mathrm{p}>0.05)$ entre mayo y octubre. Sin embargo, sí se encontraron diferencias altamente significativas $(p>0.0001)$ al comparar el largo de gametóforo entre las dos especies, tanto para mayo, como para octubre.

El ancho de gametóforos de ambas especies a lo largo de 5 sitios de colecta (Fig. 5b) fue significativamente diferente $(p<0.0002)$ para el mes de mayo, a diferencia del mes de octubre, cuando no presentaron diferencias significativas $(\mathrm{p}>0.05)$. Para ambas especies, el ancho de gametóforo fue significativamente diferente $(\mathrm{p}<0.03)$ entre fechas de colecta (mayo vs. octubre). De manera similar, el ancho del gametóforo de las dos especies fue significativamente diferente $(\mathrm{p}<0.005)$, tanto en mayo como en octubre (Fig. 5b).

El diámetro de gametangios para cada especie de Batophora no fue significativamente diferente ni por fechas, ni por sitios de colecta 
CUADRO 2

Biomasa especifica y total (por $25 \mathrm{~cm}^{2}$ ) de macroalgas recolectadas en ocho sitios en la Bahía de Chetumal en marzo de 2000

TABLE 2

Total and specific biomass (per $25 \mathrm{~cm}^{2}$ ) of macroalgae sampled in eight sites from the Chetumal Bay, during March 2000

\begin{tabular}{|c|c|c|c|c|c|c|c|c|}
\hline & \multicolumn{8}{|c|}{ SITIOS } \\
\hline ESPECIES & $\mathrm{C} 1$ & $\mathrm{C} 2$ & $\mathrm{C} 3$ & 1 & 2 & 3 & 4 & 5 \\
\hline Batophora oerstedii & & 0.82 & 3.77 & 0.22 & & 0.45 & 1.08 & 0.12 \\
\hline Bostrychia pilulifera & & 11.8 & & & & & & \\
\hline Bostrychia tenella & & & & 1.47 & & & & \\
\hline Bostrychia sp. & & & 0.49 & & 6.72 & 1.78 & & 3.59 \\
\hline Catenella caestepitosa & & & & & 2.40 & & & 7.95 \\
\hline Cladophora sp. & 0.55 & 1.10 & 0.30 & & & 2.03 & & \\
\hline Enteromorpha intestinalis & 0.03 & & & & & & & \\
\hline Enteromorpha sp. & & & & & & 0.56 & & \\
\hline Lyngbya sp. & 1.75 & & & & & & & \\
\hline Polysiphonia sphaerocarpa & & 0.27 & & & & & & \\
\hline Polysiphonia subtilissima & 2.78 & 0.22 & & & & & & \\
\hline Polysiphonia sp. & & & 4.92 & & & 1.72 & & 0.12 \\
\hline Symploca sp. & & & & & & 0.83 & & \\
\hline TOTAL & 5.11 & 14.21 & 9.47 & 1.69 & 9.13 & 7.37 & 1.08 & 11.77 \\
\hline
\end{tabular}

(Fig. 5c). Sin embargo, sí existieron diferencias altamente significativas $(\mathrm{p}<0.001)$ entre especies, ya que el diámetro de los gamentangios de $B$. oerstedii fue mayor (entre 78 y $82 \mu \mathrm{m}$ ), en comparación al de $B$. occidentalis (entre 55 y $65 \mu \mathrm{m})$ (Fig. 5c).

La biomasa promedio obtenida en marzo de 2000 (Cuadro 2) fue máxima en el sitio C2 (14.21 g) y mínima en el sitio 4 (1.08 g). El primero se encuentra ubicado cercano a descargas de aguas residuales de la ciudad de Chetumal.

La temperatura fue mayor en mayo que en octubre (Fig. 6a), mientras que la precipitación pluvial fue casi nula en mayo y máxima en julio. En octubre la precipitación pluvial fue relativamente mayor que en mayo (Fig. 6b). La dirección predominante del viento entre mayo y septiembre fue del Este, y del Norte entre octubre y noviembre. En mayo se presentó mayor velocidad del viento que en octubre (Fig. 6c).

\section{DISCUSIÓN}

El número de taxa fue bajo y poco variable en la composición de especies para los dos muestreos efectuados en 1999, lo que indica una flora pobre y un tanto estable para la Bahía de Chetumal, de manera similar a como lo informaron Solé y Vera (1997) para sitios de la costa de Venezuela.

En la época de secas se registró un mayor número de taxa (17) que en la época de lluvias (11) para el sistema estuarino de Bahía Chetumal (Cuadro 1). Esto es diferente de lo informado por Mateo-Cid y Mendoza-González (1991), Mendoza-González y Mateo-Cid (1992) 

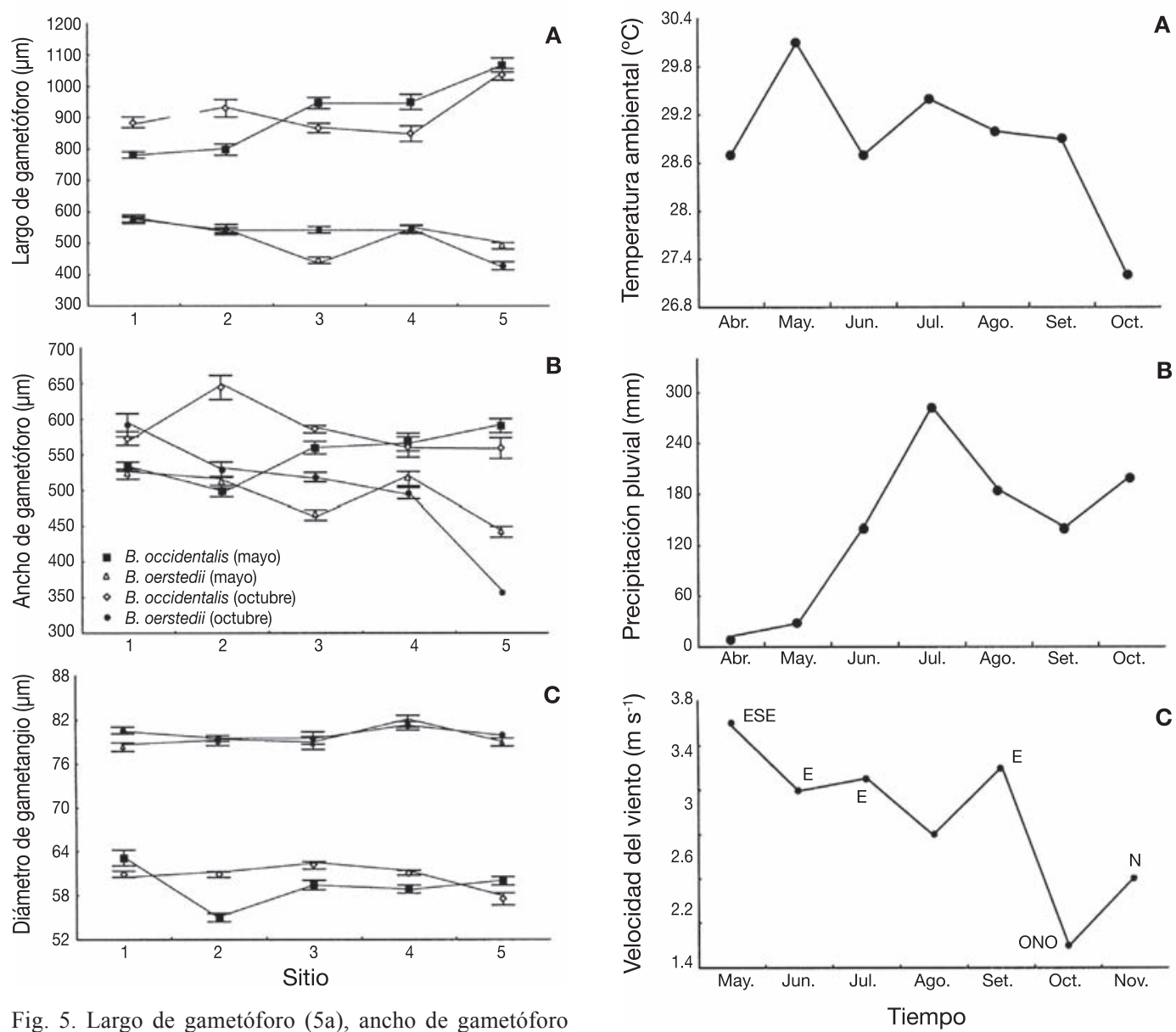

Fig. 5. Largo de gametóforo (5a), ancho de gametóforo (5b) y diámetro de gametangio (5c) de Batophora occidentalis y $B$. oerstedii en cinco sitios de la Bahía de Chetumal, durante mayo y octubre de 1999. Todas las medidas en $\mu \mathrm{m}$.

Fig. 5. Length of gametophore (5a), width of gametophore (5b) and diameter of gametangia (5c) of Batophora occidentalis and $B$. oerstedii in five sites from the Chetumal Bay, during May and October 1999. All measurements in $\mu \mathrm{m}$.

y Díaz-Martín et al. (1998), quienes para aguas marinas del Caribe mexicano encontraron un mayor número de especies en la temporada climática de lluvias que en secas. Por otra parte, las pocas especies (4) de Phaeophyta registradas para la bahía de Chetumal (HuertaMúzquiz et al. 1987, este estudio) resaltan la escasa presencia de esta División en lagunas

Fig. 6. Valores promedio de temperatura $\left({ }^{\circ} \mathrm{C}, 6 \mathrm{a}\right)$, precipitación pluvial $(\mathrm{mm}, 6 \mathrm{~b})$ y velocidad $\left(\mathrm{m} \mathrm{s}^{-1}\right)$ y dirección dominante del viento (6c) en la ciudad de Chetumal, Quintana Roo, en 1999. E= Este, ESE= Este-Sureste, N= Norte, $\mathrm{ONO}=$ Oeste-Noroeste.

Fig. 6. Mean values of temperature $\left({ }^{\circ} \mathrm{C}, 6 \mathrm{a}\right)$, rainfall $(\mathrm{mm}$, $6 \mathrm{~b}$ ), and velocity $\left(\mathrm{m} \mathrm{s}^{-1}\right)$ and dominant direction of the wind (6c) from the Chetumal city, State of Quintana Roo, during 1999. $E=$ East, $E S E=$ East-Southeast, $N=$ North, $\mathrm{ONO}=$ West-Northwest.

costeras (Espinoza-Avalos 1993, Díaz-Martín et al. 1998).

Los agrupamientos de macroalgas por sitio (con similitud $>0.5$ ) fueron diferentes para las dos épocas climáticas (Fig. 2 y 3), y el sitio 1 quedó aislado del resto. Éste es el que recibe una mayor cantidad de aguas residuales de la ciudad de Chetumal. Coincidentemente, 
para las colectas de 1999 sólo en los sitios 1 y 4 se presentaron especies de Enteromorpha (Cuadro 1), que se han registrado en ambientes contaminados por materia orgánica (Morand y Briand 1996). En esos dos sitios también ocurrieron las mayores concentraciones de materia orgánica en el sedimento (LlanezBaeza y Salazar-Vallejo 2000). Cabe destacar, sin embargo, que el origen de esa materia orgánica puede ser diferente: por contaminación de descargas municipales para el primer sitio, y por procesos naturales (producto de la descomposición de materia orgánica proveniente de Laguna Guerrero), para el segundo sitio.

La composición de especies de macrofitas no reflejó la contaminación orgánica a lo largo de cinco sitios, como en otros trabajos donde se reporta una reducción de especies (Neverauskas 1987, Cecere et al. 1991). Sin embargo, en el sitio con desagües (C1) predominaron especies que otros autores (Murray y Littler 1978, Druehl 1981, Levine 1984, Guangzhou 1987, Morand y Brand 1996) registran en sitios contaminados (Enteromorpha y Polysiphonia). Por otra parte, Batophora spp. es dominante en Bahía Chetumal (Axis-Arroyo et al. 1998, Cuadro 1), excepto en el sitio C1. Esto es, algunas macroalgas indicadoras de contaminación se presentan principalmente donde ocurren descargas directas de aguas residuales, mientras que Batophora spp. es eliminada.

Los nuevos registros para la Bahía de Chetumal se pueden explicar por los escasos trabajos ficoflorísticos que se han efectuado en la zona de estudio (Huerta-Múzquiz y GarzaBarrientos 1980, Huerta-Múzquiz et al. 1987).

La riqueza de la flora béntica registrada en el muestreo de mayo de 1991 fue de 25 taxa. El sitio 4 (Bacalar Chico) fue el que presentó mayor riqueza (13 taxa, Cuadro 1). Esto puede explicarse por la influencia de aguas del Mar Caribe, ya que ese sitio se encuentra ubicado en un canal de comunicación entre la Bahía de Chetumal y ese mar, con mayor número de especies algales (Littler y Littler 1997). En contraparte, el resto de los sitios, ubicados dentro de la Bahía de Chetumal, con promedio anual bajo de salinidad (13\%o, Gasca y Castellanos 1993), en general presentaron riqueza específica baja (Cuadro 1).

Es importante señalar que la distinción entre las dos especies de Batophora presentes en la Bahía de Chetumal sólo se puede efectuar cuando las plantas se encuentran en reproducción, ya que los caracteres vegetativos son indistinguibles entre $B$. oerstedii y $B$. occidentalis (Gómez-Poot et al. en prensa). Por ejemplo, la talla de ambas especies (Fig. 3) no fue significativamente diferente en las dos fechas y cinco sitios de colecta. Sin embargo, el largo de sus gametóforos (Fig. 5a) y diámetro de gametangios (Fig. 5c) fue claramente diferente entre ambas especies.

Ninguna de las mediciones vegetativas y reproductivas efectuadas en Batophora oerstedii y $B$. occidentalis indicaron influencia de la contaminación en la Bahía de Chetumal, del sitio 1 al 5. Por ejemplo, las plantas más largas de $B$. occidentalis ocurridas en mayo (Fig. 3a) se encontraron en el sitio 2, mientras que en octubre (Fig. 3b) esto ocurrió en el sitio 3. De manera similar, tallas equivalentes de las dos especies se encuentran en sitios con más influencia de contaminación orgánica (sitio 1), y en otros alejados de las fuentes de contaminación de la cuidad de Chetumal (sitios 2 y 5 , en mayo, y 2 y 4 , en octubre, por ejemplo). Conclusiones similares se obtienen al analizar las mediciones de gametóforos y gametangios (Fig. 5). Es decir, las dos especies Batophora no mostraron cambios vegetativos ni reproductivos consistentes, como los registrados en otros trabajos (Censen 1984, Levine 1984, Turubí et al. 1985, Haglund et al. 1996, Amado 1997). En otras palabras, las mediciones realizadas en $B$. oerstedii y B. occidentalis no parecen "anticipar" o dar signos de la presencia de materia orgánica, por lo que no se podrían considerar como especies indicadoras de contaminación (McCormick y Cairns 1994).

Las diferentes respuestas reproductivas de Batophora oerstedii y $B$. occidentalis en los sitos de estudio y las diferencias en sus ciclos reproductivos de plantas maduras e inmaduras 
(Fig. 4) posiblemente también originan que el reclutamiento de ambas especies ocurra en tiempos diferentes, lo cual pudiera ayudar a disminuir la competencia por espacio entre ambas. Diferencias en fenología reproductiva se han sugerido como procesos que permiten la coexistencia de plantas vasculares (Bengtsson et al. 1994), y el estudio de Marinho-Soriano et al. (1998) muestra un ejemplo de coexistencia de dos especies de Gracilaria (Rhodophyta) que presentan fenologías divergentes. De manera similar, las estrategias reproductivas de $B$. oerstedii y $B$. occidentalis probablemente ayudan a que coexistan tan cercanamente (ocupando las mismas rocas) en la Bahía de Chetumal.

\section{AGRADECIMIENTOS}

D. Esquivel-Moreno por su apoyo en la recolección de macroalgas. A la SEMARNAP por haber financiado la mayor parte del trabajo, a través del proyecto "Bioindicadores Bénticos de la Bahía de Chetumal”, y por haber proporcionado una beca de investigación a S.G.J.F.

\section{RESUMEN}

Los componentes de la flora béntica, y mediciones de partes vegetativas y reproductivas de Batophora oerstedii y $B$. occidentalis (Chlorophyta) se registraron en cinco sitios de la costa Oeste de la Bahía de Chetumal, donde se ha registrado la existencia de un gradiente de contaminación orgánica. La colecta de flora se realizó en mayo y Octubre de 1999, meses incluidos en las épocas climáticas de secas y lluvias, respectivamente. Se registraron 40 taxa de vegetación béntica, de los cuales 11 representan nuevos registros para la Bahía de Chetumal, y 6 para el Caribe Mexicano. Especies de Enteromorpha estuvieron presentes en ambientes ricos en materia orgánica, tanto de origen urbano, como natural. En los desagües de la Bahía de Chetumal existen especies indicadoras de contaminación, pero la ausencia de Batophora spp., dominante en esta laguna costera. Los caracteres morfológicos de B. occidentalis y $B$. oerstedii a lo largo de los cinco sitios de muestreo no reflejaron la presencia de contaminantes. Ambas especies presentaron diferencias significativas en su largo y ancho de gametóforos, y en el diámetro de gametangios.
Las diferencias en estrategias reproductivas probablemente ayudan a que $B$. oerstedii y $B$. occidentalis puedan coexistir cercanamente en la Bahía de Chetumal.

Palabras clave: Enteromorpha, flora béntica, contaminación, coexistencia, Caribe, Península de Yucatán.

\section{REFERENCIAS}

Aguilar-Rosas, L.E. \& I. Pacheco-Ruiz. 1989. Influencia de desechos municipales-industriales sobre macroalgas del Norte de Baja California, México. Biol. Inst. Oceonogr. Venezuela. Univ. Oriente. 28: 77-84.

Amado, G.M., C.S. Karez, L.R. Andrade, Y. YoneshigueValentin \& W.C. Pfeiffer. 1997. Effects on growth and accumulation of zinc in six seaweed species. Ecotoxicol. Environ. Safety. 37: 223-228.

Axis-Arroyo J., B. Morales-Vela, D. Torruco-Gómez \& M.E. Vega-Cendejas. 1998. Variables asociadas con el uso de hábitat del manatí del Caribe (Trichechus manatus), en Quintana Roo, México (Mammalia). Rev. Biol. Trop. 46: 791-803.

Bengtsson, J., T. Fagerström \& H. Rydin. 1994. Competition and coexistence in plant communities. TREE 9: 246250 .

Berger, S. \& M.J. Kaever.1992. Dasycladales. An Illustrated Monograph of a Fascinating Algal Order. Georg Thieme, Stuttgart, Alemania vii+ 247 p.

Chavira-Martínez, D., J. Briceño-González, A. Negroe, J.A. Pérez-Cetina, T. Sánchez- Cabrera \& R. Hoil. 1992. Diagnóstico de la calidad del agua en la Bahía de Chetumal, Q. Roo. Avacient. 3: 16-31.

Cecere, E., M. Cormaci \& G. Furnari. 1991. The marine algae of Mar Piccolo, Taranto (Southern-Italy): A reassessment. Bot. Mar. 34: 221-227.

Díaz-Martín, M.A., E. Torres-Mejía \& J. Espinoza-Avalos. 1998. Lista de algas del Área de Protección Yum Balam, Quintana Roo, México. Rev. Biol. Trop. 46: 487-492.

Dreckmann, K.M. 1998. Clasificación y Nomenclatura de las Macroalgas Marinas Bentónicas del Atlántico Mexicano. Depto. de Hidrobiología UAM, México. $140 \mathrm{p}$.

Druehl, L.D., 1981. Geographical distribution. p. 306-325. In L.S. Lobban \& M.J. Wynne (eds.). The Biology of Seaweeds. Blackwell, Oxford, Inglaterra. 
Espinoza-Avalos, J. 1993. Macroalgas marinas del Golfo de California. p. 328-357. In S.I. Salazar-Vallejo \& N.E. González (eds.). Biodiversidad Marina y Costera de México. CONABIO-CIQRO, Chetumal, México.

Gasca, R. \& I. Castellanos. 1993. Zooplancton de la Bahía de Chetumal, Mar Caribe, México. Rev. Biol. Trop. 41: 619-625.

Gómez-Poot, J.M., J. Espinoza-Avalos \& S.G. JiménezFlores. 2002. Vegetative and reproductive characteristics of two species of Batophora (Chlorophyta: Dasycladaceae) from Chetumal Bay, Quintana Roo, Mexico. Bot. Mar. 45: 189-195.

Guangzhou, Y.B., 1987. Ulva lactuca (Chlorophyta, Ulvales) in Hong Kong intertidal waters. Its nitrogen and phosphorus contents and its use as a bioindicator of eutrophication. Asian Mar. Biol. 4: 97-102.

Haglund, K., M. Björklund, S. Gunnare, A. Sandberg, U. Olander \& M. Pedersén. 1996. New method for toxicity assessment in marine and brackish environments using the macroalga Gracilaria tenuistipitata (Gracilariales, Rhodophyta). Hydrobiology. 326/327: 317-325.

Huerta-Múzquiz, L. \& A. Garza-Barrientos. 1980. Contribución al conocimiento de la zona Sur del litoral de Quintana Roo, México. An. Esc. Nac. Cienc. Biol. Méx. 23: 25-44.

Huerta-Múzquiz, L., A.C. Mendoza-González \& L.E. Mateo-Cid. 1987. Avance sobre un estudio de las algas marinas de la Península de Yucatán. Phytology. 62: $22-53$.

Jensen, A., 1984. Marine ecotoxicological tests with seaweeds. p. 181-193. In G. Persoone, E. Jaspers \& C. Claus (eds.). Ecotoxicological Testing for the Marine Environment. Vol. 1. State Univ. Ghent and Inst. Mar. Scient. Res., Bredene, Bélgica.

Joly, A.B. 1967. Generos de algas marinhas da costa Atlantica latinoamericana. Universidade de Sâo Paulo, Sâo Paulo, Brasil. 461 p.

Levine H.G. 1984. The use of seaweeds for monitoring coastal waters. p. 189-210. In Algae as Ecological Indicators. Academic, Londres, Inglaterra.

Littler, D.S. \& M.M. Littler. 1997. An illustrated marine flora of the Pelican Cays, Belize. Bull. Biol. Soc. Wash. 9: 1-149.

Llanes-Baeza, C.A. \& S.I. Salazar-Vallejo. 2000. Evaluación de los mejillones Mytillopsis sallei (Récluz, 1849) y Brachidontes exustus (Linné, 1758) como bioindicadores en la Bahía de Chetumal, Quintana Roo. p. 28-51. In Bioindicadores Bénticos de la Bahía de
Chetumal. Informe final. SEMARNAP-ECOSUR, México.

Marinho-Soriano, E., T. Laugier \& M.L. Casabianca. 1998. Reproductive strategy of two Gracilaria species, $G$. bursa-pastoris and G. gracilis, in a Mediterranean Lagoon (Thau, France). Bot. Mar. 41: 559-564.

Mateo-Cid, L.E. \& A.C. Mendoza-González.1991. Algas marinas bénticas de Cozumel, Quintana Roo, México. Act. Bot. Méx. 16: 57-87.

McCormick, P.V. \& J. Cairns. 1994. Algae as indicators of environmental change. J. Appl. Phycol. 6: 509-526.

Mendoza-González, A.C. \& L.E. Mateo-Cid. 1992. Algas marinas bentónicas de Isla Mujeres, Quintana Roo, México. Act. Bot. Mex. 19: 37-61.

Morand, P. \& X. Briand. 1996. Excessive growth of macroalgae: a symptom of environmental disturbance. Bot. Mar. 39: 491-516.

Murray, S.N. \& M.M. Littler. 1978. Patterns of algal succession in a perturbated marine intertidal community. J. Phycol. 14: 506-512.

Neverauskas, V.P. 1987. Accumulation of periphyton biomass on artifitial substrates deployed near a sewage sludge outfall in South Australia. Estuar. Coast. Shelf. Sci. 25: 509-517.

Ortega, M.M. 1984. Catálogo de Algas Continentales Recientes de México. Instituto de Biología. UNAM, México. 566 p.

Ortegón-Aznar, I. 1997. Estudios de integración ficoflorística de tres lagunas costeras de la Península de Yucatán. Tesis Profesional. Facultad de Ciencias, UNAM, México. 163 p.

Ortiz H., M.C. \& R. Sáenz M. 1995. Monitoreo de la contaminación de la Bahía de Chetumal, Quintana Roo. Centro de Investigación de Q. Roo (CIQROO) y Consejo Nacional de Ciencia y Tecnología (CONACYT). 51 p.

Ortiz, M.C. \& R. Sáenz. 1997. Detergents and orthophosphates inputs from urban discharges to Chetumal Bay Quintana Roo, México. Bull. Environ. Contam. Toxicol. 59: 486-491.

Ortiz H., M.C. \& R. Sáenz M. 1999. Effects of organic material and distribution of fecal coliforms in Chetumal Bay, Quintana Roo, México. Environ. Monitor. Assess. 55: 423-434.

Ross, P. \& M.E. DeLorenzo. 1997. Sediment contamination problems in the Caribbean islands: Research and regulations. Environ. Toxicol. Chem. 16: 52-58. 
Solé-S., M.A. \& B. Vera-V. 1997. Caracterización de las macroalgas marinas bénticas en la región ChirimenaPunta Caimán, Edo. Miranda, Venezuela. Caribb. J. Sci. 33: 180-190.

Taylor, W.R. 1960. Marine Algae of the Eastern Tropical and Subtropical Coasts of the Americas. The University of Michigan, Ann Arbor, Michigan, EEUU. 9: 125-203.
Thursby, G.B., R.L. Steele \& M.E. Kane. 1985. Effect of organic chemicals on growth and reproduction in the marine red alga Champia parvula. Environ. Toxicol. Chem. 4: 797-805.

Wynne, M.J. 1998. A checklist of benthic marine algae of the tropical and subtropical Western Atlantic. First revision. Nova Hedwigia, Beiheft 116: 1-155. 
